Eveline Oliverra de CAstro'

MaRia RItA de FiguerRedo

LEMOS BORTOLOTTO²

Marcelo ZUGaiB ${ }^{3}$

\title{
Sepse e choque séptico na gestação:
} manejo clínico

\author{
Sepsis and septic shock during pregnancy: \\ clinical management
}

\section{Revisão}

Palavras-chave Sepse

Choque séptico Complicações na gravidez Mortalidade materna Complicações infecciosas na gravidez

Keywords

Sepsis

Shock, septic Pregnancy complications Maternal mortality Pregnancy complications, infectious
Correspondência:

Maria Rita de Figueiredo Lemos Bortolotto Rua Vieira de Moraes, 420, cito. 36 CEP 046170-000 - São Paulo/SP E-mail: ritalb@uol.com.b

Recebido

$19 / 11 / 08$

\section{Resumo}

A sepse é uma das principais causas de morte materna, estando relacionada a infecções de origem obstétrica laborto infectado, corioamnionite, infecção puerperal) ou não-obstétricas (resultando de infecções que acometem outros sítios). Esta revisão tem por objetivo descrever os mecanismos envolvidos na fisiopatologia desta entidade e atualização da abordagem clínica da sepse recomendada em diretrizes internacionais ("early goal-directed therapy" - ressuscitação precoce, ou tratamento precoce guiado por metas), bem como chamar a atenção para a influência do estado gravídico tanto no quadro clínico, quanto no manejo terapêutico dos quadros sépticos.

\section{Abstract}

Sepsis is one of the main causes of maternal death, being related to infections from obstetric origin (infected abortion, chorioamnionitis, puerperal infection) or non-obstetric (resulting from infections which occur in other areas). This review aims at describing the mechanisms involved in the physiopathology of this entity and at updating the clinical approach to sepsis, recommended in international guidelines (early goal-directed therapy - precocious resuscitation, or precocious treatment guided by goals), as well as at calling attention to the influence of pregnancy both in the clinical manifestation and in the therapeutic management of septic conditions.
Clínica Obstétrica do Hospital das Clínicas da Faculdade de Medicina da Universidade de São Paulo - USP - São Paulo (SP) Brasil.

' Residente do Departamento de Obstetrícia e Ginecologia da Faculdade de Medicina da Universidade de São Paulo - USP São Paulo (SP), Brasil.

${ }^{2}$ Assistente da Clínica Obstétrica do Hospital das Clínicas da Faculdade de Medicina da Universidade de São Paulo - USP São Paulo (SP), Brasil.

${ }_{3}^{3}$ Professor Titular da Disciplina de Obstetrícia da Faculdade de Medicina da Universidade de São Paulo - USP - São Paulo (SP), Brasil. 


\section{Introdução}

Sepse é uma das grandes causas de morbimortalidade em todo o mundo. Nos Estados Unidos, é a causa de 2 a $11 \%$ de todas as admissões hospitalares ${ }^{1,2}$. Estudos brasileiros mostram diferenças regionais nas taxas de mortalidade, o que se justifica pela heterogeneidade populacional, bem como pelas diferenças no acesso à saúde ${ }^{3}$. Trata-se também de uma das cinco principais causas de mortalidade materna ${ }^{2}$. Entretanto, alguns fatores contribuem para melhor prognóstico da sepse em obstetrícia, como o sítio de infecção mais comum ser a pelve (região passível de intervenção cirúrgica) e a maior sensibilidade dos principais microorganismos à antibioticoterapia de amplo espectro ${ }^{4}$.

Esta revisão objetiva atualizar o obstetra quanto às últimas diretrizes recomendadas no tratamento da sepse e choque séptico, visando ao reconhecimento e tratamento precoce destas entidades, bem como ao auxílio ao médico intensivista na condução de gestantes e puérperas nestas condições.

Quadro 1 - Definições dos termos empregados nesta revisão

\begin{tabular}{|c|c|}
\hline Termo & Definiç̣̃o \\
\hline Bacteremia & $\begin{array}{l}\text { Presença de bactérias viáveis na corrente sangǘnea. Pode não ter } \\
\text { relevância clínica e sua presença não é suficiente para o diagnóstico } \\
\text { de sepse. }\end{array}$ \\
\hline Sepse & $\begin{array}{l}\text { Síndrome clínica caracterizada por resposta deletéria do hospedeiro a } \\
\text { um processo infeccioso; infecção acompanhada de resposta inflamatória } \\
\text { sistêmica. }\end{array}$ \\
\hline $\begin{array}{l}\text { Síndrome da resposta } \\
\text { inflamatória sistêmica } \\
\text { (SIRS) }\end{array}$ & $\begin{array}{l}\text { Presença de dois ou mais critérios abaixo: } \\
\text { - } \quad \text { Temperatura }>380 \text { C ou }<360 \mathrm{C} \\
\text { - } \quad \text { Freqüência cardíaca }>90 \text { bpm (desde que o paciente não esteja } \\
\text { em uso de drogas cronotrópicas negativas) } \\
\text { - } \quad \text { Freqüência respiratória }>20 \text { irpm ou PaCO2 }<32 \mathrm{mmHg} \\
\text { - } \quad \text { Leucócitos }>12.000 / \mathrm{mm} 3 \text { ou }<4.000 / \mathrm{mm} 3 \text { ou }>10 \% \\
\text { de formas jovens }\end{array}$ \\
\hline Sepse grave & $\begin{array}{l}\text { Sepse associada à disfunçáo de um ou mais órgãos (SNC, renal, } \\
\text { pulmonar, hepática, cardíaca, coagulopatia, acidose metabólica) }\end{array}$ \\
\hline Choque séptico & Sepse com hipotensão refratária à ressuscitação volêmica * \\
\hline \multicolumn{2}{|c|}{$\begin{array}{l}\text { Adaptado de: Levy MM, Fink MP, Marshall JC, Abraham E, Angus D, Cook D, et al. } 2001 \text { SCCM/ESICM/ } \\
\text { ACCP/ATS/SIS International Sepsis Definitions Conference. Crit Care Med. 2003;31(4):1250-65. }\end{array}$} \\
\hline \multicolumn{2}{|c|}{$\begin{array}{l}\text { Irpm=incursões respiratórias por minuto; bpm=batimentos por minuto; } \\
\mathrm{SNC}=\text { sistema nervoso central. }\end{array}$} \\
\hline \multicolumn{2}{|c|}{$\begin{array}{l}\text { *Os parâmetros que definem hipotensão refratária são: pressão sistólica } \\
<90 \mathrm{mmHg} \text {; pressão arterial média < } 65 \mathrm{mmHg} \text {; diminuição de } 40 \mathrm{mmHg} \text { na } \\
\text { pressão sistólica, comparada à pressão basal do indivíduo, e não-resposta à } \\
\text { infusão de fluidos }(20 \text { a } 40 \mathrm{ml} / \mathrm{kg})^{5} \text {. }\end{array}$} \\
\hline
\end{tabular}

Quadro 2 - Principais microorganismos relacionados à sepse na gestação

\begin{tabular}{lc}
\hline Grupo & Microorganismo \\
\hline Aeróbios Gram-negativos & $\begin{array}{c}\text { Enterococos (E. coli, Klebsiella pneumoniae, Enterobacter, } \\
\text { Proteus, Serratia) e Pseudomonas aeruginosa }\end{array}$ \\
Aeróbios Gram-positivos & $\begin{array}{c}\text { Estreptococos (S. agalactiae, S. pyogenes) e Enterococos (S. } \\
\text { faecalis, S. faecium) }\end{array}$ \\
Anaeróbios & Peptococcus, Peptostreptococcus, Clostridium perfringens, \\
Bacteroides, Fusobacterium
\end{tabular}

Adaptado de: Cabar FR. Choque séptico. In: Zugaib M. Bittar RE, editores. Protocolos assistenciais da Clínica Obstétrica da FMUSP. 3a ed. São Paulo: Atheneu; 2007. p. 649-56.6.

\section{Definições e conceitos}

Os termos relacionados à sepse foram utilizados arbitrariamente por muitos anos, o que dificulta comparações e investigações epidemiológicas. A definição dos termos atualmente empregados (bacteriúria, sepse, síndrome da resposta inflamatória sistêmica, sepse grave e choque séptico) foi publicada somente em 2003 e encontra-se no Quadro $1^{5}$.

\section{Microbiologia e fatores associados}

Os quadros infecciosos mais associados à sepse podem ser subdividos em não-obstétricos e obstétricos. O primeiro grupo engloba pneumonia adquirida em comunidade, infecções do trato urinário, apendicite, colecistite, Vírus da Imunodeficiência Humana (HIV) e malária.

Já as principais causas obstétricas relacionam-se à gestação (corioamnionite, tromboflebite pélvica séptica, aborto infectado); ao parto (endometrite pós-parto, infecção de episiotomia, infecção de parede ou uterina pós-cesárea) e à realização de procedimentos invasivos (infecção pós-cerclagem ou pós-amniocentese, fasciíte necrotizante) ${ }^{1}$. Os quadros de sepse de origem obstétrica são, em geral, de origem polimicrobiana, sendo as bactérias Gram-negativas as mais freqüentes ${ }^{2,6}$. Os principais microorganismos estão listados no Quadro 2.

\section{Fisiopatologia}

O conhecimento sobre a fisiopatologia da sepse vem crescendo muito nos últimos anos, mas ainda existem muitas questões a serem respondidas. A gravidade do quadro depende de inúmeros fatores, dentre os quais a virulência do organismo agressor e fatores relacionados ao hospedeiro, tais como idade, genética, sítio da infecção e presença de comorbidades ${ }^{1}$.

A ativação da resposta imune ocorre a partir da liberação de "padrões moleculares associados ao patógeno", ou seja, endotoxinas da parede celular de Gram-negativos (lipopolissacarídeos) e exotoxinas de alguns Gram-positivos (peptideoglicanas, ácido lipoteicóico). Tais componentes interagem com receptores específicos na superfície de monócitos, denominados "toll-like receptors", resultando em uma complexa cascata de eventos que envolvem a ativação de neutrófilos, macrófagos e linfócitos e a liberação de mediadores inflamatórios ${ }^{1-3}$.

As células $\mathrm{T} \mathrm{CD}_{4}$ ativadas podem produzir citocinas com atividade pró-inflamatória (TNF- $\alpha$, interferon- $\gamma$, IL-2), sendo denominadas células Th1, ou citocinas com atividade antiinflamatória (IL-4, IL-10), sendo denominadas células Th2. Os fatores que determinam a predominância de Th1 ou Th2 na sepse permanecem desconhecidos, mas provavelmente são influenciados pelo tipo de patógeno, tamanho do inóculo e local da infecção. Inicialmente, a 
sepse é caracterizada pelo aumento de mediadores inflamatórios, porém, com a progressão do quadro, há uma mudança para um estado de imunossupressão ${ }^{7}$.

A reação inflamatória inicial leva à geração de radicais livres e óxido nítrico sintase. A produção de óxido nítrico ativa a guanilato ciclase solúvel e gera GMP cíclico, causando desfosforilação da miosina na parede muscular endotelial e, conseqüentemente, vasodilatação e aumento da permeabilidade vascular ${ }^{4,8}$. Estudos recentes mostram também que o óxido nítrico em excesso inibe a função mitocondrial da musculatura esquelética, o que dificulta a oxigenação adequada deste tecido ${ }^{3}$.

A resposta hemodinâmica acontece precocemente; a redução da resistência vascular leva a queda da pressão arterial, o que provoca taquicardia por estímulo aos barorreceptores. Com isso, há aumento do débito cardíaco e ativação do sistema renina-angiotensina ${ }^{1}$. Apesar dos altos níveis de angiotensina-2 e norepinefrina detectáveis na circulação de pacientes com sepse, é conhecido ainda que o óxido nítrico promove hiperpolarização da membrana celular dos miócitos que compõem a camada muscular endotelial, impedindo a ação dos primeiros ${ }^{8}$.

A fase inicial da sepse (fase "quente") é caracterizada por pele quente e seca (devido à vasodilatação periférica), febre, hipotensão, taquicardia, confusão mental, ansiedade e taquidispnéia. Com a progressão do quadro (fase "fria"), a hipoperfusão resulta em acidose láctica, piora da perfusão tecidual (levando a cianose de extremidades) e disfunção orgânica ${ }^{6}$.

As citocinas pró-inflamatórias têm importante efeito sobre a cascata de coagulação. $O$ TNF- $\alpha$ age na superfície endotelial, induzindo a liberação de fator tecidual (o primeiro passo da via extrínseca), culminando na produção de trombina, que, por sua vez, catalisa a transformação de fibrinogênio em fibrina. Além disso, o TNF- $\alpha$, em associação ao IL-1, eleva a produção de inibidor do ativador de plasminogênio-1, um potente inibidor da fibrinólise ${ }^{2-4}$.

Em situações fisiológicas, quando a trombina se complexa à trombomodulina, há ativação da proteína $\mathrm{C}$. Esta forma ativa, juntamente com a proteína $S$, inativa os fatores Va e VIIIa da cascata da coagulação, exercendo efeito anticoagulante ${ }^{3}$. Foi observado em pacientes com sepse um aumentado consumo desses e de outros fatores anticoagulantes (antitrombina e inibidor do fator tecidual) e baixas taxas de síntese. O resultado final é o estado pró-coagulante, que pode culminar com o quadro de coagulação intravascular disseminada ${ }^{2,4}$. A deposição de fibrina na microvasculatura é um dos principais mecanismos de falência orgânica, graças à oclusão da mesma com conseqüente déficit de oxigenação ${ }^{3}$.

A ativação de citocinas com propriedades antiinflamatórias surge, então, como resposta a esse processo.
É observada concomitantemente a ocorrência de outros fenômenos que compõem o quadro de imunossupressão: anergia e apoptose de células T CD4, células B e dendríticas e perda da expressão do complexo MH II pelos macrófagos ${ }^{7}$. É acreditado que o desbalanço dos mecanismos pró e antiinflamatório seja responsável por dificultar o restabelecimento do paciente, tornando-o susceptível a outras infecções ${ }^{1,2}$.

\section{Sepse e alterações fisiológicas da gestação}

$\mathrm{Na}$ gestação e puerpério, algumas manifestações do quadro de sepse podem ser modificadas pelas alterações sistêmicas e locais observadas neste período:

\section{Sistema cardiovascular}

Durante a gestação, ocorre diminuição da resistência vascular periférica (por influência de mediadores como prostaciclina, óxido nítrico e hormônios gestacionais) e aumento da freqüência cardíaca, com conseqüente queda da pressão arterial e aumento do débito cardíaco. Isso pode não somente mascarar sinais iniciais de sepse, como também piorar a hipoperfusão normalmente associada ao quadro ${ }^{1}$.

Como já foi observado, na sepse ocorre perda do tônus vasomotor e disfunção contrátil do miocárdio. Como o aumento do débito cardíaco na gestação é o maior responsável pela manutenção da pressão arterial, a sobrecarga na função cardíaca materna pode levar à instabilidade hemodinâmica rapidamente ${ }^{2}$.

Ocorre aumento do volume plasmático (de 30 a 50\%) e do volume globular (em até $25 \%$ ), o que, a partir do sexto mês de gestação, reflete na queda da hemoglobina e do hematócrito, constituindo a chamada "anemia fisiológica da gestação" " Na sepse grave e choque séptico, tal condição pode piorar a oferta de oxigênio aos tecidos. A hipoperfusão uterina e fetal decorrente deste fenômeno pode levar a acidose metabólica fetal ${ }^{2}$.

\section{Função pulmonar}

As alterações no sistema respiratório têm por objetivo aumentar a oferta de oxigênio para mãe e feto e incluem o aumento do volume corrente associado à redução do volume residual, sem alterações na capacidade pulmonar total - com isso, a expiração materna se torna mais completa e há maior quantidade de ar a ser inspirado. Ocorre aumento do volume-minuto em 30 a $40 \%{ }^{1,9}$.

A progesterona promove o estímulo ao centro respiratório, levando à diminuição da $\mathrm{PaCO}_{2}$; como mecanismo compensatório há diminuição dos valores do bicarbonato sérico. A hiperventilação fisiológica cria maior gradiente de oxigênio do feto para a mãe. Durante a sepse, porém, isso dificulta uma resposta rápida à acidose metabólica e prejudica a oxigenação ${ }^{1,9}$. 
Com a hemodiluição fisiológica, há redução na concentração de proteínas séricas e diminuição da pressão coloidosmótica. $\mathrm{Na}$ sepse, tais mudanças favorecem a ocorrência de edema pulmonar ${ }^{1,2}$. A síndrome da angústia respiratória sistêmica é uma grave complicação da sepse, a qual apresenta uma taxa de mortalidade entre gestantes de 30 a $60 \%{ }^{2}$. Ocorre geralmente nas primeiras 24 a 48 horas do quadro e é caracterizada por taquidispnéia, hipoxemia aguda $\left(\mathrm{PaO}_{2} / \mathrm{FIO}_{2}<200 \mathrm{mmHg}\right)$, infiltrado pulmonar bilateral e ausência de evidência de falência cardíaca esquerda (pressão de capilar pulmonar $\leq 8 \mathrm{mmHg})^{1}$.

\section{Função renal}

Foi observado na grande maioria das gestantes, dilatação ureteropiélica (principalmente à direita) secundária à obstrução mecânica pelo útero e ao relaxamento da musculatura lisa ureteral ${ }^{1,9}$. Isso leva à estase urinária, com risco aumentado para infecções de trato urinário de uma forma geral, as quais se não tratadas podem evoluir para pielonefrite ${ }^{1}$.

O fluxo plasmático renal e a taxa de filtração glomerular se elevaram na gestação, resultando em níveis reduzidos de uréia e creatinina séricos ${ }^{1,9}$. Dessa forma, níveis considerados normais fora do período gravídico podem se relacionar a comprometimento renal leve na sepse. A falência renal na sepse é relacionada à necrose tubular aguda, cujos principais mecanismos de lesão são o de isquemia-reperfusão, decorrente do aumento do tônus simpático, e a deposição de microtrombos nos glomérulos, secundária ao estado pró-coagulante da gestação e da sepse per $s e^{1,2}$.

\section{Aparelho gastrintestinal}

Ocorre diminuição do tônus muscular em todo o trato gastrintestinal durante a gravidez, o que leva ao refluxo gastroesofágico, retardo do esvaziamento gástrico e trânsito intestinal lento ${ }^{1,9}$. Tais mudanças, associadas a fenômenos da sepse - aumento da permeabilidade da mucosa gástrica e lesão mucosa secundária a hipoperfusão - facilitam a ocorrência de translocação bacteriana (e, portanto, uma piora do quadro clínico), além de aumentar o risco de pneumonia aspirativa ${ }^{1,2}$.

A gestação promove ainda mudanças na composição da bile, aumentando o risco de colelitíase; durante a sepse, ocorre também aumento da produção de citocinas inflamatórias pelas células de Kupfer (estimuladas tanto pela isquemia quanto por endotoxinas), levando a colestase, hiperbilirrubinemia e icterícia ${ }^{2}$.

\section{Coagulação}

As modificações gravídicas nos componentes do sistema de coagulação objetivam assegurar o domínio das perdas hemorrágicas no sítio placentário. Estas ocorrem a partir do primeiro trimestre, concomitantemente à elevação do estrogênio e da progesterona, que são os possíveis elementos causais desta modificação?. Ocorre elevação dos fatores VII, VIII, IX, X, XII, fator de Von Willebrand e fibrinogênio e redução da proteína $\mathrm{S}$, além de redução da atividade fibrinolítica decorrente do aumento de inibidor do ativador de plasminogênio 1 e $2^{2}$. O estado pró-trombótico gestacional eleva substancialmente o risco de coagulação intravascular disseminado em pacientes com sepse.

\section{Manejo clínico da paciente com sepse}

Apesar do grande avanço na compreensão da imunopatologia da sepse, o conhecimento a respeito do tratamento progride lentamente. As diretrizes para tratamento de tais condições permanecem em contínuo aprimoramento, uma vez que sua fisiopatologia ainda não está totalmente esclarecida. Em 2004, foi publicado o primeiro Surviving Sepsis Campaign guidelines for managent of severe sepsis and septic shock, por um grupo de experts representando 11 organizações, sendo as primeiras diretrizes aceitas internacionalmente para o tratamento da sepse e choque séptico. A publicação da última atualização do referido artigo $^{10}$, que contou com a participação de 55 experts internacionalmente reconhecidos, possui data de 2008.

O reconhecimento e a instituição precoce do tratamento da sepse e seus espectros são cruciais para o prognóstico materno e fetal. A prioridade do tratamento deve ser dada à gestante, especialmente na fase inicial, uma vez que o bem-estar fetal depende principalmente da melhora dos parâmetros maternos ${ }^{1,2,4}$. Se a gestante está hemodinamicamente instável, a realização do parto pode aumentar a chance de morte, exceto em casos em que o foco infeccioso é intra-uterino ${ }^{1}$.

As diretrizes para o tratamento da sepse, descritas a seguir, são baseadas em evidências que variam de altas a muito baixas e determinam seu grau de recomendação ${ }^{11}$. As estratégias terapêuticas foram desenvolvidas para a ressuscitação inicial (zero a seis horas) e manutenção ( $>6$ horas). $\mathrm{O}$ conjunto das condutas recomendadas no manejo clínico da sepse e seus espectros compõem a early goal-directed therapy (tratamento precoce guiado por metas). A seguir, serão descritas as etapas desta abordagem terapêutica, discutindo as particularidades exercidas pelo estado gravídico. Durante as primeiras seis horas de tratamento (ressuscitação inicial), os parâmetros a serem atingidos são ${ }^{10}$ : pressão venosa central (PVC): 8 a $12 \mathrm{mmHg}$; pressão arterial média: $\geq 65 \mathrm{mmHg}$; débito urinário: $\geq 0,5 \mathrm{~mL} / \mathrm{kg} / \mathrm{h}$ e saturação venosa de $\mathrm{O}_{2}$ (veia cava superior): $\geq 70 \%$.

Em situações normais, a PVC das gestantes é maior que a população não-grávida. Contudo, não existem estudos acerca da PVC em gestantes com sepse, o que 
dificulta a validação dessa medida no grupo em questão ${ }^{1}$. No caso de gestantes em ventilação mecânica, para facilitar o retorno venoso, é possível elevar o valor da PVC para 12 a $15 \mathrm{mmHg}$ e posicionar a paciente de forma a evitar compressão da veia cava inferior pelo útero ${ }^{1}$.

As estratégias descritas a seguir visam ao cumprimento das metas acima estabelecidas. A resposta clínica na gestante pode ser avaliada pela pressão arterial, freqüência cardíaca, saturação de oxigênio, débito urinário e pelo status fetal ${ }^{1}$.

\section{Diagnóstico}

É recomendada a obtenção de pelo menos duas hemoculturas (uma de sangue periférico e uma proveniente de cateter central, a menos que este tenha sido inserido há menos de 48 horas), além de culturas de prováveis sítios infecciosos (urina, liquor, secreções do trato respiratório, pontas de cateteres) antes do início da antibioticoterapia ${ }^{4,10}$. Em gestantes com suspeita de corioamnionite, deve ser considerada a realização de amniocentese ${ }^{1}$.

Vale ressaltar a importância dos exames de imagem como métodos complementares para o diagnóstico topográfico da infecção. A paciente só deve ser transportada para o local do exame se estiver hemodinamicamente estável. Em caso contrário, pode dispor de exames realizáveis à beira do leito, como radiografia e ultra-sonografia ${ }^{1,10}$.

\section{Antibioticoterapia}

A introdução de antibióticos deve ser iniciada na primeira hora após o reconhecimento da sepse grave e choque séptico; o atraso nessa medida promove aumento mensurável da mortalidade ${ }^{10,12}$. É recomendado que o tratamento seja iniciado com uma ou mais drogas de amplo espectro e que atinjam concentrações adequadas nos prováveis focos de infecçãa ${ }^{10}$. A escolha da droga deve maximizar os efeitos para a gestante e minimizar danos ao feto, em geral, se possível evitar tetraciclinas, fluoroquinolonas, cloranfenicol e estolato de eritromicina ${ }^{1,2}$.

Uma vez estabelecido o diagnóstico do sítio da infecção e identificado o agente, é recomendável estreitar o espectro de ação da antibioticoterapia, objetivando maior efetividade local, menor índice de resistência bacteriana e menor suscetibilidade a organismos multirresistentes (tais como espécies de Candida, Clostridium difficile e Enterococcus faecium resistente à vancomicina $)^{10}$.

Entretanto, devem ser observados os casos de gestantes com provável foco infeccioso obstétrico, pois, devido ao caráter polimicrobiano dessas infecções, pode não ser adequado estreitar o espectro terapêutico, mesmo após identificação do agente em cultura. Para estes tipos de casos, geralmente foi optada a associação de ampicilina (ou penicilina), gentamicina e metronidazol.
A duração do tratamento, de forma geral, é de sete a dez dias, podendo ser individualizada de acordo com a resposta clínica. Por fim, caso seja verificado que o choque tem origem não-infecciosa, é recomendado que a antibioticoterapia seja suspensa, devendo evitar as conseqüências do tratamento com agentes de amplo espectro, como visto acima ${ }^{10}$.

\section{Controle do foco infeccioso}

Uma vez elucidado o foco infeccioso, devem ser verificadas a possibilidade de intervenção local, ou seja, drenagem de abscessos; desbridamento de tecido necrótico ou mesmo remoção cirúrgica do órgão acometido, se possível nas primeiras seis horas do diagnóstico. Também é recomendado que a intervenção seja a menos invasiva possível $^{10}$.

No caso de pacientes com corioamnionite associada à sepse grave ou choque séptico, o parto deve ser o mais precoce possível, independentemente da idade gestacional. A via de parto é de determinação obstétrica, sendo o parto vaginal o mais recomendável; no caso de cesáreas de urgência (devido à instabilidade hemodinâmica ou sofrimento fetal não-responsivo à ressuscitação materna) é indicada a realização de anestesia geral ${ }^{1}$. O parto não é indicado para fins terapêuticos se o foco infeccioso não for a gestação ${ }^{2}$.

O controle do foco pode apresentar algumas complicações, tais como sangramento, fistulização e lesão inadvertida de órgãos, tendo que atentar mais para as mesmas na evolução clínica ${ }^{10}$.

\section{Administração de fluidos}

Em pacientes hipovolêmicos, como medida inicial é recomendada a administração de fluidos por via endovenosa, iniciando com $1.000 \mathrm{~mL}$ de cristalóide ou 300 a $500 \mathrm{~mL}$ de colóide nos primeiros 30 minutos $^{10}$. Não há evidências de superioridade entre as soluções acima apresentadas. O objetivo dessa terapêutica é atingir os parâmetros vitais citados anteriormente.

\section{Vasopressores}

Caso a reposição volêmica não seja suficiente para a manutenção da estabilidade hemodinâmica da paciente, é necessária a administração de vasopressores - sendo as de primeira escolha a norepinefrina e a dopamina. Estes devem ser administrados por meio de cateter central e com monitorização invasiva da pressão arterial, não havendo contra-indicação ao seu uso na gestação. $\mathrm{O}$ objetivo do tratamento é manter a pressão arterial média igual ou superior a $65 \mathrm{mmHg}^{1,10}$.

A dopamina, quando em baixas doses $(<10 \mathrm{mg} / \mathrm{kg} /$ min), eleva a pressão arterial média e o débito cardíaco, devido ao seus efeitos inotrópico e cronotrópico positivos. É 
particularmente útil em pacientes com comprometimento da função sistólica, porém é mais arritmogênico ${ }^{4,10}$. A norepinefrina, por sua vez, tem maior efeito vasoconstritor, sendo mais efetivo na reversão da hipotensão em pacientes com choque séptico ${ }^{10}$. A vasopressina induz vasoconstrição por ação nos receptores V1 na camada muscular endotelial ${ }^{12}$ e não há estudos relatando seu uso na gestação.

$\mathrm{O}$ uso de vasopressores requer atenção à monitorização fetal, pois tanto a dopamina quanto a noradrenalina podem reduzir o fluxo sangüíneo útero-placentário ${ }^{1,4}$.

\section{Corticosteróides}

Em pacientes com choque séptico, foi observada insuficiência adrenal relativa, situação que eleva substancialmente a mortalidade e a necessidade de suporte inotrópico ${ }^{4}$. Sprung et al. ${ }^{13}$ avaliaram, no estudo Corticosteroid Therapy of Septic Shock (CORTICUS), a eficácia e a segurança da administração de hidrocortisona em pacientes com choque séptico. O estudo mostrou que a hidrocortisona diminui o tempo para a reversão do choque, porém não reduz a taxa de mortalidade (o que se deve ao aumento da taxa de superinfecção, inclusive novos episódios de choque séptico, no grupo submetido ao tratamento).

Esse mesmo estudo mostrou que o efeito da corticoterapia no choque séptico independe da resposta do paciente ao teste de estimulação com corticotropina. Isso permite concluir que tal teste não deve ser usado para selecionar os pacientes que devem ser submetidos ao tratamento com corticosteróide. Ainda se sabe que os níveis de cortisol e a resposta à corticotropina são diferentes na paciente grávida, o que também advoga em favor da terapia empírica ${ }^{1}$. O uso da hidrocortisona não é contra-indicado na gestação.

Dessa forma, é sugerida a administração de corticosteróides (hidrocortisona $300 \mathrm{mg} / \mathrm{d}$ ou fludrocortisona $50 \mathrm{mcg} / \mathrm{d}$ ) somente para pacientes com choque séptico associado a baixa resposta à reposição volêmica e ao uso de drogas vasoativas. Na ausência de choque, os mesmos não devem ser administrados ${ }^{10}$.

Em casos de provável parto prematuro com feto viável, pode ser indicada a administração de betametasona (12 mg IM duas vezes, com intervalo de 24 horas entre as mesmas) para maturação pulmonar fetal ${ }^{1}$.

\section{Proteína C ativada humana recombinante}

$\mathrm{O}$ uso da proteína $\mathrm{C}$ ativada no tratamento de pacientes com sepse é sugerido nos casos de disfunção de múltiplos órgãos. A proteína $C$ ativada humana recombinante aumenta o risco de sangramento e é contra-indicada em pacientes com sangramento ativo ou procedimento cirúrgico recente $(<30 \text { dias })^{1,4,10}$, o que, de certa forma, limita o seu uso em Obstetrícia.

\section{Uso de hemoderivados}

Se a paciente não apresenta resposta adequada à expansão volêmica e a saturação venosa de $\mathrm{O}_{2}$ não atinge $70 \%$ durante as primeiras seis horas pode ser realizada (em associação ao uso de vasopressores) a transfusão de concentrado de hemácias, objetivando níveis de hematócrito iguais ou superiores a $30 \%$ (nível de evidência $B)^{1,2,4}$.

Uma vez estabelecidos os parâmetros de perfusão adequada, quando os níveis de hemoglobina forem inferiores a 7,0 g/dL, é recomendada a administração de concentrado de hemácias para manter tais níveis entre 7,0 e 9,0 g/dL ${ }^{10}$. Tais valores podem ser insuficientes para fornecer oxigenação adequada ao feto, devendo, portanto, utilizar o perfil biofísico-fetal como medida indireta do fluxo úteroplacentário e da oxigenação sangüínea materna ${ }^{1}$.

É sugerido que plasma fresco congelado não seja administrado para pacientes sem sangramento ativo e sem programação de procedimentos invasivos ${ }^{10}$. A administração de plaquetas deve ser feita quando a contagem for menor que $5.000 / \mathrm{mm}^{3}$, se não houver risco de sangramento. Se, por outro lado, houver risco aparente de sangramento, o nível de corte para transfusão sobe para $30.000 / \mathrm{mm}^{3}$; por fim, para procedimentos cirúrgicos ou invasivos, é recomendado ${ }^{10}$ que a contagem plaquetária seja igual ou superior a $50.000 / \mathrm{mm}^{3}$.

\section{Ventilação mecânica}

A ventilação mecânica apresenta basicamente dois benefícios: melhora a troca de gases e diminui o esforço respiratório. Sua maior indicação é a insuficiência respiratória aguda (IRpA), geralmente presente no curso do choque séptico ${ }^{14}$. A IRpA caracteriza-se por sintomas (dispnéia, prostração, dor torácica e cefaléia), sinais (taqui ou bradipnéia, tiragens, cianose, agitação ou rebaixamento do nível de consciência) e alterações gasométricas - sendo classificada em tipo I ou hipoxêmica $\left(\mathrm{PaO}_{2}<60 \mathrm{mmHg}\right.$ ou $\left.\mathrm{SaO}_{2}<90 \%\right)$ e tipo II ou hipercápnica $\left(\mathrm{PaCO}_{2}>45\right.$ $\mathrm{mmHg}$ ou elevação $>10 \mathrm{mmHg}$ com $\mathrm{pH}<7,35)$. Uma vez identificada a IRpA, o suporte ventilatório deve ser iniciado precocemente ${ }^{14}$.

Como parâmetros de ventilação mecânica são recomendados ${ }^{10}$ : volume corrente de $6 \mathrm{ml} / \mathrm{kg}$ (peso presumido); pressão de platô $\leq 30 \mathrm{cmHg}$; hipercapnia permissiva (objetivando manter padrões de volume corrente e pressão de platô seguros) e uso da pressão expiratória final (PEEP) $>5 \mathrm{cmH}_{2} \mathrm{O}$, a fim de evitar colapso alveolar à expiração.

Como visto anteriormente, na gestação é observada elevação da $\mathrm{PaCO}_{2}$ e redução dos níveis séricos de bicarbonato; essa alcalose respiratória compensada é essencial para a difusão de $\mathrm{O}_{2}$ para o feto, no qual foi possível observar um mecanismo inverso ao da mãe (elevação do bicarbonato sérico). A hipercapnia permissiva, quando 
prolongada, promove um desbalanço nesse mecanismo, com conseqüente acidose metabólica fetal ${ }^{1}$.

Pacientes em ventilação mecânica devem ter seu leito elevado de $30 \mathrm{a} 45^{\circ}$, a fim de minimizar os riscos de aspiração e pneumonia (lembrando que a alimentação enteral não deve ser dada com o leito a $0^{\circ}$ ). $\mathrm{O}$ uso de cateter de artéria pulmonar atualmente não é recomendado, pois os parâmetros por ele fornecidos são conflitantes e não existem medidas comprovadas para melhorá-los ${ }^{10}$.

Em pacientes ventilados mecanicamente e com lesão pulmonar, após a estabilização hemodinâmica, a administração de fluidos deve ser criteriosa, a fim de diminuir o ganho de peso e, assim melhorar a oxigenação ${ }^{10}$.

Em face de uma resposta clínica adequada, a extubação deve ser considerada quando presentes os seguintes critérios: resolução da causa da insuficiência respiratória; suspensão da sedação até Glasgow > 10, com impulso respiratório presente; estabilidade hemodinâmica, sem uso de drogas vasoativas (exceto dobutamina); $\mathrm{pH}$ entre 7,30 e 7,60, com eletrólitos normais; estabilização hídrica (balanço hídrico zero ou negativo), baixos parâmetros ventilatórios e necessidade de $\mathrm{O}_{2}$ que pode ser suprida por máscara ou cânula nasal.

A extubação deve ser precedida de desmame da ventilação mecânica, que se traduz pelo processo de redução do suporte ventilatório oferecido ao paciente. $\mathrm{O}$ método mais comum de desmame é o tubo T. Por meio dessa peça, acoplada entre o tubo traqueal e o sistema de ventilação, é verificada a existência de respiração espontânea por intervalos progressivamente maiores. O teste pode ser realizado várias vezes no mesmo dia ou uma vez, diariamente. Se positivo por 30 minutos ou mais, indica possibilidade de extubação ${ }^{15}$.

\section{Sedação, analgesia e bloqueio neuromuscular}

De forma geral, é recomendada que a sedação seja realizada de forma contínua, com interrupções diárias ("despertares") ou sob a forma de bolus intermitentes ${ }^{10}$.

Tanto os analgésicos quanto os sedativos e bloqueadores neuromusculares podem atravessar a barreira placentária e causar diminuição da variabilidade da freqüência cardíaca e dos movimentos corpóreos fetais; assim, seu uso deve ser criterioso e mínimo. Atualmente, a administração rotineira de bloqueadores neuromusculares é contra-indicada ${ }^{1,10}$.

\section{Controle glicêmico e suporte nutricional}

É recomendado que os níveis glicêmicos sejam monitorizados a cada duas horas e mantidos entre $80 \mathrm{e}$ $110 \mathrm{mg} / \mathrm{dL}$ nas gestantes. Essa medida é eficaz na redução da taxa de infecções nosocomiais e da necessidade de suporte ventilatório ${ }^{2,4,10}$.

O suporte nutricional também deve ser individualizado. Associado ao aumento da necessidade energética da paciente com sepse é necessário considerar a elevação da necessidade calórica da gestante (cerca de $300 \mathrm{kcal} / \mathrm{dia}$ no segundo e terceiro trimestres $)^{16}$.

\section{Profilaxia de trombose venosa profunda}

Pacientes em unidade de terapia intensiva são pacientes de risco para trombose venosa profunda (TVP). Dessa maneira, a menos que existam contra-indicações (trombocitopenia, coagulopatia grave ou hemorragia intracraniana recente) deve ser feita a profilaxia com heparina não-fracionada ou de baixo peso molecular, inclusive durante a gestaçãa ${ }^{2,10}$. Nos casos em que houver contraindicação ao uso de heparina, é recomendada a introdução de medidas não-farmacológicas, tais como compressão intermitente de panturrilhas. Todos os pacientes com sepse grave e choque séptico, por serem considerados de alto risco para TVP, devem ser tratados profilaticamente com heparina e medidas não-farmacológicas ${ }^{10}$.

\section{Profilaxia de úlceras de stress}

Pacientes com sepse grave ou choque séptico geralmente apresentam condições que favorecem a formação de úlceras gástricas, como ventilação mecânica, hipotensão e coagulopatia; assim sendo, a profilaxia de úlceras de stress é recomendada e deve ser feita com bloqueadores $\mathrm{H} 2$ ou inibidores da bomba de prótons ${ }^{2,10}$.

\section{Administração de bicarbonato}

O uso de bicarbonato de sódio no tratamento da acidose láctica (induzida por hipoperfusão) não é recomendado por falta de evidências ${ }^{10}$.

\section{Conduta obstétrica}

Decisões quanto ao momento do parto são sempre polêmicas. De maneira geral, se o feto não é viável, o objetivo do tratamento é a recuperação materna; já em gestações de termo, o parto pode ser realizado assim que a paciente estiver estável. Entre esses extremos é situada a zona de maior conflito, na qual pesam o diagnóstico infeccioso, o prognóstico materno e a decisão da família ${ }^{1}$.

\section{Conclusões}

Sepse durante a gestação é um evento dramático e ainda de alta mortalidade, cuja fisiopatologia permanece em contínua investigação. As alterações fisiológicas da gestação podem mascarar ou mesmo agravar o quadro clínico, logo cabe ao obstetra conhecê-las para realizar o diagnóstico precoce, o qual é elemento crucial para o prognóstico materno e fetal.

Após o diagnóstico deve ser procedido à estabilização hemodinâmica da paciente, utilizando os parâmetros fornecidos pelo tratamento precoce guiado por metas. Algumas 
das principais medidas devem ser implementadas já na primeira hora, como a introdução de antibioticoterapia de amplo espectro e a ressuscitação volêmica, enquanto outras podem ser introduzidas no decorrer das primeiras seis horas, a depender da resposta clínica da paciente (uso de vasopressores e transfusão de sangue, entre outros). $\mathrm{Na}$ sepse grave e choque séptico, é fundamental que a paciente seja internada em unidade de terapia intensiva.

A identificação do foco infeccioso é imprescindível e deve ser feita a distinção entre as causas obstétricas (geralmente de caráter polimicrobiano, sensível a antibioticoterapia de amplo espectro e passível de intervenção cirúrgica) das causas não-obstétricas. Se a causa infecciosa for relacionada à gestação, torna-se necessária a realização do parto; nas demais, podem ser considerados procedimentos pouco ou menos invasivos para eliminação do foco.

Por fim, a participação ativa do obstetra junto ao médico intensivista é fundamental, a fim de elucidar as particularidades do tratamento da sepse grave e choque séptico na paciente grávida, estabelecer relação com a paciente e informá-la sobre o status fetal e, por fim, manter contato com os familiares e decidir, juntamente com eles, o melhor momento para intervenções.

\section{Referências}

1. Guinn DA, Abel DE, Tomlinson MW. Early goal directed therapy for sepsis during pregnancy. Obstet Gynecol Clin North Am. 2007;34(3):459-79,xi.

2. Fernández-Pérez ER, Salman S, Pendem S, Farmer JC. Sepsis during pregnancy. Crit Care Med. 2005;33(10 Suppl):S286-93.

3. Silva E, Passos RDH, Ferri MB, Figueiredo LFP. Sepsis: from bench to bedside. Clinics. 2008;63(1):110-20.

4. Sheffield JS. Sepsis and septic shock in pregnancy. Crit Care Clin. 2004;20(4):651-60.

5. Levy MM, Fink MP, Marshall JC, Abraham E, Angus D, Cook D, et al. 2001 SCCM/ESICM/ACCP/ATS/SIS International Sepsis Definitions Conference. Crit Care Med. 2003;31(4):1250-6.

6. Cabar FR. Choque séptico. In: Zugaib M, Bittar RE, editores. Protocolos assistenciais da Clínica Obstétrica da FMUSP. 3a ed. São Paulo: Atheneu; 2007. p. 649-56.

7. Hotchkiss RS, Karl IE. The pathophysiology and treatment of sepsis. N Engl J Med. 2003;348(2):138-50.

8. Landry DW, Oliver JA. The pathogenesis of vasodilatory shock. N Engl J Med. 2001;345(8):588-95.

9. Rezende J, Montenegro CAB. Obstetrícia fundamental. 9a ed. Rio de Janeiro: Guanabara Koogan; 2003. p. 73-87.
10. Dellinger RP, Levy MM, Carlet JM, Bion J, Parker MM, Jaeschke $R$, et al. Surviving Sepsis Campaign: international guidelines for management of severe sepsis and septic shock: 2008. Intensive Care Med. 2008;34(1):17-60.

11. Bensenõr IM, Lotufo PA. Epidemiologia: abordagem prática. São Paulo: Sarvier; 2005. p. 1-19.

12. Parrilo JE. Septic shock - vasopressin, norepinephrine, and urgency. N Engl J Med. 2008;358(9):954-5.

13. Sprung $C L$, Annane D, Keh D, Moreno R, Singer M, Freivogel $K$, et al. Hydrocortisone therapy for patients with septic shock. N Engl J Med. 2008;358(2):111-24.

14. Slutsky AS. Mechanical ventilation. American College of Chest Physicians' Consensus Conference. Chest. 1993;104(6): 1833-59.

15. Jubran A, Tobin MJ. Methods of weaning from mechanical ventilation [ Internet]. 2007 [cited 2008 Oct 12]. Available from: http://www.uptodate.com/patients/content/topic. do? topicKey $=\sim y T p o X D g . J z 00 A$

16. Bittar RE, Fazio ES. Aspectos nutricionais. In: Zugaib M, Bittar $R E$, editores. Protocolos assistenciais da Clínica Obstétrica da FMUSP. 3a ed. São Paulo: Atheneu; 2007. p. 3-12. 